\title{
Percepción del perfil del turista para el aprovechamiento de los atractivos turísticos: Caso Cantón Pasaje, El Oro (Ecuador)
} Perception of the tourism profile for the
exploitation of tourist attractions: Case of
Pasaje Canton, El Oro (Ecuador)

Dayanara Sandoval A. ${ }^{1}$ Oscar Ordoñez C. ${ }^{2}$, Mauricio Noblecilla G. ${ }^{3}$

Resumen: Ecuador es uno de los destinos más visitados en la actualidad por sus atractivos turísticos, paisajes naturales y culturales. En los últimos años el turismo ha venido dando varios cambios que han ayudado a mejorar la calidad de vida de las personas y comunidades existentes; la actividad turística se ha convertido en el principal ingreso del país, siendo ésta una clave para el desarrollo de la economía nacional. El presente estudio tuvo por objetivo identificar los diferentes perfiles que cuentan los turistas para un buen aprovechamiento de los atractivos turísticos del cantón Pasaje, Provincia de EI Oro. Para el desarrollo de esta investigación se empleó un método descriptivo, utilizando el instrumento metodológico de tipo encuesta, la cual se aplicó a los pobladores de la parroquia con una muestra de 395 personas. Los resultados obtenidos muestran que el cantón Pasaje no dispone de buena publicidad para dar a conocer los diferentes atractivos turísticos, es por ese motivo que no cuenta con mucha demanda turística, para el buen desarrollo económico y aprovechamiento de los atractivos existentes del sitio.

Palabras clave: Atractivos turísticos, calidad de vida, de- manda turística, atractivos.

Abstract: Ecuador is currently one of the most frequently visited destinations for its tourist attractions, natural landscapes and cultural scenes. In recent years, tourism has undergone various changes that have helped improve the quality of life of the people and existing communities; tourist activity has become the country's main income, and this is key to the growth of the national economy. This study sought to identify different tourist profiles in order to develop the tourist attractions in Pasaje Canton, El Oro Province. A descriptive method was used, employing a survey, which was administered to the residents of the parish with a sample of 395 people. The results show that the Pasaje Canton does not have good publicity to make the different tourist attractions known, which is why it does not have much tourism demand for good economic development and exploitation of the site's existing attractions.

Key words: Tourist attractions, quality of life, tourism demand, attractions.

(Presentado: 28 de agosto de 2017. Aceptado: 5 de noviembre de 2017)

\footnotetext{
${ }^{1}$ Administración de Hotelería y Turismo, Universidad Técnica de Machala, Ecuador. E-mail: dlsandoval_estqutmachala.edu.ec

${ }^{2}$ Magíster en Planificación y Gestión de Proyectos Agroturísticos y Ecológicos, Universidad Agraria del Ecuador. Docente Ocasional, Universidad Técnica de Machala, Ecuador. E-mail: osordonezQutmachala.edu.ec

${ }^{3}$ Doctor en Ciencias Administrativas, Universidad Nacional Mayor de San Marcos, Perú. Docente Titular Auxiliar, Universidad Técnica de Machala, Ecuador. E-mail: msnoblecillaqutmachala.edu.ec.
} 


\section{INTRODUCCIÓN}

Ecuador es uno de los destinos más visitados en la actualidad por sus atractivos turísticos, paisajes naturales y culturales. En los últimos años el turismo ha venido dando varios cambios que han ayudado a mejorar la calidad de vida de las personas y comunidades existentes; la actividad turística se ha convertido en el principal ingreso del país, siendo ésta una clave para el desarrollo de la economía nacional. Las diferentes modalidades de viaje permiten que los turistas visiten lugares que realmente les agrade, los haga sentir cómodos y los haga regresar a vivir más experiencias; el que las personas se sientan satisfechas en un sitio ayuda al progreso del turismo y patrimonio del mismo, también es turismo las visitas a familiares o amigos, los negocios, la religión, la cultura y otros motivos.

Se define por atractivo al conjunto de bienes y servicios, materiales o inmateriales, estén o no institucionalizados bajo alguna categoría patrimonial, cuya característica definitoria es que poseen cierto grado de atractividad para el visitante. Así como en el proceso de patrimonialización el poder político juega un rol fundamental, para este caso, la característica de "turístico" implica que los segmentos de la demanda jueguen también un papel esencial en esta suerte de proceso de "activación turística" o "turistificación" (Bustos, 2001). Según Romero (2015), el cantón Pasaje, Provincia de El Oro (Ecuador), tiene un impresionante potencial para desarrollar el turismo de naturaleza; es ese valor agregado que lo distingue de los otros cantones de la Provincia, sus encantos naturales, paisajes, ríos, gastronomía y clima.

Este trabajo fue realizado es en el cantón Pasaje, el cual posee muchos atractivos turísticos interesantes y novedosos para que los turistas lo visiten, pero existe un inadecuando aprovechamiento por parte de la población existente, usando los atractivos solo fines de semana y feriado, durante el cual ellos proceden a vender comida para ganar dinero extra, pero no lo ven con fines turísticos. Los lugares más conocidos del cantón son: Balneario "Las Dos Bocas", "La Cocha"; La Laguna del Amor; Cascada Cerro Azul, y Cascadas o Pailones de Casacay, entre otros. La finalidad de este trabajo es identificar los diferentes perfiles con que cuentan los turistas para un buen aprovechamiento de los atractivos turísticos del cantón Pasaje.

El cantón Pasaje es una entidad territorial sub-nacional ecuatoriana, de la Provincia de El Oro. Se ubica en la Re- gión Costa. Su cabecera cantonal es la ciudad de Pasaje, lugar donde se agrupa gran parte de su población total. La ciudad y el cantón Pasaje, al igual que las demás localidades ecuatorianas, se rige por una municipalidad según lo estipulado en la Constitución Política Nacional. La municipalidad de Pasaje es una entidad de Gobierno seccional que administra el cantón de forma autónoma al Gobierno Central. La municipalidad está organizada por la separación de poderes de carácter ejecutivo representado por el Alcalde, y otro de carácter legislativo conformado por los miembros del concejo cantonal. El Alcalde es la máxima autoridad administrativa y política del cantón. Es la cabeza del cabildo y representante del municipio. El cantón se divide en parroquias que pueden ser urbanas o rurales $y$ son representadas por las Juntas Parroquiales ante el Municipio de Pasaje. Las parroquias urbanas son Ochoa León, Bolívar, Loma de Franco, y Tres Cerritos. Las parroquias rurales son Buenavista, Cañaquemada, La Peaña, Uzhcurrumi, El Progreso, y Casacay.

Las principales fuentes de ingresos económicos de la población agrupa las actividades económicas provenientes de la agricultura $80 \%$, banano, cacao y cítricos; pecuaria $10 \%$; ganadería, avícola; minera $7 \%$, explotación de material pétreo en cerros y ríos y turismo 3\%. El cantón Pasaje cuenta con una envidiable infraestructura natural para la adecuación de balnearios y otros sitios de esparcimiento, convirtiéndose el turismo en otras de las fuentes de recursos de ingreso al cantón. Para ello se requiere de inversión del Estado y del sector privado para desarrollar este potencial recurso natural.

\section{MARCO REFERENCIAL}

Los viajes han sido frecuentemente objeto de inspiración para escritores y cronistas que han ido dejando la huella de su experiencia viajera en el desarrollo de sus textos. En ellos se suele alternar lo real con lo imaginario y es esa una de las principales fortalezas que puede tener un trabajo literario para alcanzar el éxito. La constante intromisión de lo real en lo ficticio y viceversa permite la construcción de incesantes imaginarios sociales en determinados lugares y momentos. Las creaciones literarias transportan al lector a una realidad que sólo existe o se configura en su cabeza. Con el auge y desarrollo del turismo literario, que es una rama del turismo cultural, se va a permitir, de múltiples maneras, que el lector complemente en el mundo 
real lo que experimentó en sus lecturas, por ejemplo visitando los lugares donde discurre la trama de una novela. En este artículo se analiza, por un lado, el turismo literario como factor de creación de nuevos imaginarios sociales y, por otra parte, se construye un perfil del turista literario en España que nos permita identificar cuáles son sus características comunes. Para conseguir este fin se revisó bibliografía específica sobre el tema tratado, se recurrió a organismos culturales que aportaron datos sobre el visitante y su experiencia viajera y se incorporó una encuesta como técnica para obtener información (Pérez-Redondo, 2017).

El turismo es una fuente importante de crecimiento económico local. Esto implica que es muy relevante conocer los gustos, preferencias y hábitos de viaje del turista, de manera de incorporar criterios técnicos en la planificación de la inversión pública y privada, y en la creación, innovación o especialización de productos turísticos. La planta hotelera de Otavalo, provincia de Imbabura, Ecuador, juega un rol importante en la actividad turística de la zona. Sin embargo, existe un alto nivel de desconocimiento de la situación actual de la demanda turística. Es por ello que en esta investigación se determinó el perfil del turista que ocupa la planta hotelera de Otavalo, de forma de contribuir al diseño de la oferta turística. Se utilizó un diseño no experimental de corte transversal. La población objeto de estudio estuvo conformada por los representantes de los establecimientos de alojamiento (Censo) y por los turistas nacionales y extranjeros $(n=380)$ del primer semestre de 2015. También se realizaron entrevistas en profundidad a los principales gestores públicos de turismo. Los principales resultados muestran que predomina la presencia de turistas extranjeros (61\%). El turista nacional se caracteriza por tener educación superior, entre 26 y 35 años de edad (34\%), soltero (48\%), del tipo profesional universitario (57\%), con ingresos mensuales menores a 800 dólares (62\%), y que realiza turismo dos o tres veces al año, la mayoría procede de las ciudades de Cuenca, Guayaquil y Esmeraldas (70\%), el turista extranjero también posee educación superior, hombre (53\%), entre 18 y 25 años de edad (55\%), soltero (73\%), del tipo profesional universitario (60\%), con ingresos mensuales entre 3.000 y 5.000 dólares ( $41 \%)$, y que realiza turismo más de cuatro veces al año (54\%). La mayoría procede de Estados Unidos, Canadá, Alemania y España (58\%). Una importante implicancia de esta investigación es que se debe mejorar Otavalo como destino turístico, a través del rescate, visibilización y revalorización del patrimonio cultural material e inmaterial para la realización de rutas turísticas (Realpe \& Benítez, 2015).
En los últimos años se ha incrementado la actividad turística en varios países, explicado en gran parte por el creciente interés en el denominado "turismo orientado a la naturaleza”. Este último incluye el turismo natural, turismo comunitario, turismo cultural, de aventura y el agroturismo. En este contexto adquiere especial relevancia dentro de la industria turística el conocimiento de la demanda turística. En esta investigación se estudió el perfil de la demanda turística de la modalidad "turismo de naturaleza" en la ciudad de Puyo, provincia de Pastaza-Ecuador. La metodología incluyó indicadores como género, edad, nivel de educación, motivo de viaje, recomendaciones del lugar, y medios publicitarios para la visita hacia tres emprendimientos de turismo de naturaleza: Jardín Botánico Las Orquídeas, Parque Etnobotánica Omaere y Cavernas de Fátima.

Se aplicó una metodología de investigación de carácter descriptiva, exploratoria y bibliográfica, analizadas cualitativa y cuantitativamente mediante la ficha de recopilación de información e información secundaria. Se trabajó con información del feriado de Semana Santa 2016, considerando una muestra de 67 turistas. Un 52\% de los visitantes corresponden al género masculino, las edades fluctúan entre 26 a 36 años $(53,7 \%$ ) y entre 37 a 46 años $(25,4 \%)$, la mayoría son turistas nacionales $(79,1 \%)$ de las provincias de Tungurahua y Pichincha, la mayor cantidad de los visitantes tienen un nivel académico de tercer nivel $(60,1 \%)$. El principal motivo fue por realizar turismo $(85,1 \%)$ y la totalidad de los visitantes conocieron los emprendimientos de turismo de naturaleza a través de Internet. Una importante conclusión es que en los tres emprendimientos incluidos en el estudio es necesario realizar publicidad en diferentes medios de comunicación (Corral et al., 2016).

En la última década, el turismo rural se ha convertido en una de las principales actividades económicas de la mayoría de las zonas rurales españolas. Un factor clave en el desarrollo de esta actividad ha sido la evolución que ha sufrido la demanda del sector turístico. Sin embargo, es muy poca la información acerca de los consumidores de turismo rural. En este trabajo mediante la estimación de unos modelos logit se determinó el perfil del turista que con una mayor probabilidad elige una determinada zona dentro de una región rural de oferta turística homogénea, cómo es la que constituye el noroeste de la región de Murcia (Albaladejo \& Díaz, 2003).

El Festival Internacional de las Culturas Pirineos Sur es un 
festival consolidado y de éxito. Pese a ello, su organizador, la Diputación Provincial de Huesca, necesitaba conocer con detalle el perfil del público asistente, el grado de fidelidad que genera y hasta qué punto supone un recurso turístico para la zona. Para cumplir dichos objetivos se llevó a cabo una encuesta in situ a 664 personas. Los resultados pusieron de manifiesto que la edad influye tanto en las actividades a las que se asiste como en el alojamiento elegido y el gasto. El grado de fidelidad resultó elevado. El Festival es claramente un recurso turístico de gran importancia para el Valle de Tena, lugar de celebración del evento (Barles et al., 2012).

La comuna de Montañita se encuentra ubicada en la Parroquia de Manglar Alto, provincia de Santa Elena en Ecuador, es un destino turístico de sol y playa, donde la práctica del surf es considerada uno de sus principales atractivos. Este trabajo analizó el perfil de los turistas extranjeros que visitan Montañita, así como las motivaciones que los llevan a visitar el destino y la percepción de satisfacción de los servicios turísticos ofertados en este balneario. La recolección de la información se realizó in situ. Los resultados sugieren evidencia importante acerca del perfil del turista que visita Montañita, hombres y mujeres llegan al destino de forma variada procedentes de varios continentes, en su gran mayoría son solteros, las motivaciones más valoradas fueron disfrutar del sol y la playa, seguida de descansar, lo que evidencia que la playa se convierte en el mayor atractivo turístico de este lugar. Las variables más valoradas en la satisfacción de los servicios hoteleros fue la ubicación y en los servicios de restaurantes fue la calidad de las comidas y bebidas. Los resultados obtenidos servirán para que los prestadores de servicios turísticos diseñen productos y ofrezcan servicios acorde a la demanda (Carvache et al., 2017).

El interés por la población mayor en diversas áreas de la economía y de la sociedad ha ido en aumento en los últimos años a raíz del proceso de envejecimiento demográfico que están experimentando las regiones más desarrolladas a nivel mundial, especialmente el viejo continente y particularmente España. Más allá de los costos asociados a la vejez, la población mayor se presenta como un mercado de especial relevancia para un gran número de sectores por su elevado poder adquisitivo, principalmente a través de la generación baby-boom. El sector turístico se perfila como uno de los mayores beneficiarios de ese proceso como consecuencia de los cambios en los estilos de vida de la población mayor en la actualidad, más orien- tada al disfrute del ocio que las generaciones anteriores. Por todo ello, este estudio presenta un acercamiento a los patrones de comportamiento turístico de los mayores de 55 años en España en lo que respecta a la tendencia, la motivación y las características del viaje de este colectivo (Losada et al., 2014).

Esta investigación caracteriza la oferta turística y el perfil sociocultural de los empresarios turísticos del Borde Costero de La Araucanía. Para ello se analizó la oferta turística de acuerdo a lo propuesto por Leiva (1997), haciendo énfasis en las cuatro comunas que forman parte de este territorio que presenta atractivos naturales y culturales relevantes alcanzando un total de 140 . Junto con ello, se analizó el perfil sociocultural a través de la noción de habitus planteada por Bourdieu \& Waquant (2005) que permitió reconocer dos perfiles de empresarios turísticos, uno con bajo nivel de experticia en el rubro (dados los indicadores de educación y pobreza) y cuya elección de la actividad descansa en los esfuerzos realizados desde la institucionalidad; y otro que, a partir del etnoturismo, ha consolidado una oferta turística de nivel internacional, asociadas a otros oferentes turísticos de la región de La Araucanía (Escalona et al., 2012).

Los estudios sobre turismo cultural han arrojado una serie de tipologías y perfiles sobre el turista de ese segmento que se han dado por determinantes. Sin embargo, en los últimos tiempos, las especificaciones sobre las variantes existentes dentro del turismo cultural hacen que dichos perfiles puedan no ser muy útiles a la hora de definir y conceptualizar este tipo de turismo. Para comprobar este vacío, se utilizó una festividad religiosa en Patate, zona central de la sierra ecuatoriana de los Andes, muy popular en todo el país, para medir la fiabilidad de los estándares de los perfiles turísticos. Para ello, se realizó una encuesta a los turistas religiosos que acuden las celebraciones del señor del terremoto sobre el perfil y los patrones elegidos para el estudio. Las conclusiones indican que el perfil de ambos tipos de turista es distinto, y que las mediciones sobre el perfil del turista cultural deben modificarse y ampliarse en este caso (Lavín et al., 2017).

La isla de Cozumel recibe más de dos millones de turistas al año, principalmente de los países vecinos del Norte. Si bien las investigaciones realizadas por la federación establecen diferencias geográficas, las motivaciones de los visitantes pueden ser distinguidas al examinar su capital simbólico, que permite descifrar el perfil de los turistas 
internacionales que arriban a la ínsula. Para determinar lo anterior fue necesario estudiar sus capitales económico, social, cultural y simbólico, lo cual, en la práctica turística de los individuos, se expresa de manera diferenciada en la elección de las actividades o la motivación para visitar cualquier destino. Por medio de la aplicación de encuestas en las salas del Aeropuerto Internacional de Cozumel, y de un análisis deductivo, se detectaron las particularidades de los turistas. El análisis indica que el turista internacional que visita Cozumel convive y experimenta sensaciones de satisfacción y bienestar al relacionarse con los residentes del destino, tanto en su rol de prestadores de servicios turísticos como de nativos interactuando en el mismo espacio social (Anaya \& Pelafox, 2010).

El objetivo del presente artículo es analizar los aspectos claves que definen el perfil del turismo en Andalucía, tales como las características sociodemográficas de los turistas, sus preferencias y motivaciones, o el gasto que realizan en sus viajes (Álvarez, 2012). Las motivaciones y actitud de los turistas en la actualidad están permitiendo el desarrollo de nuevos productos y destinos que permiten satisfacer las nuevas demandas de los viajeros. En este artículo presentamos una investigación centrada en el turismo del vino, en concreto la denominación de origen de Jerez Sherry. Esta denominación gira entorno a una ruta del vino cuyas bodegas reciben alrededor de 500.000 visitas anualmente. Asimismo, presentamos los resultados obtenidos de un trabajo de campo llevado a cabo dentro de las propias bodegas donde se ha realizado una encuesta a los turistas para conocer sus motivaciones y percepciones. Los principales resultados de esta investigación nos muestran el elevado grado de satisfacción de los turistas respecto a la bodega que han visitado, así como la interrelación existente entre el vino, la gastronomía local y el creciente interés de los viajeros por todo lo relacionado con la cultura del vino (López et al., 2012).

En 2014 las Islas Canarias recibieron 11 millones y medios de turistas extranjeros y Andalucía 8 millones y medio, significando respectivamente crecimientos interanuales del $17,7 \%$ y $13,1 \%$. Estos datos confirman un crecimiento sostenido en la llegada de turistas internacionales en los últimos años. En su mayoría, hablamos de turistas en busca del producto "sol y playa", aunque la tendencia ha progresado hacia una demanda multimotivacional y en consecuencia hacia una oferta más diversificada. La potenciación de la oferta cultural de los destinos consolidados busca maximizar los impactos positivos del turismo a través de la desestacionalización de los flujos turísticos y la revitalización de los centros históricos.

En este contexto, presentamos dos estudios en los que analizamos a partir de casi 1.000 encuestas presenciales, el perfil del turista cultural en dos ciudades españolas declaradas Patrimonio de la Humanidad por la UNESCO: San Cristóbal de la Laguna (Tenerife-Islas Canarias) y Córdoba (Andalucía). Ambas ciudades tienen en común, entre otras cuestiones, que se han consolidado como destino de "excursionistas" (visitantes que permanecen menos de 24 horas) pero no tanto de "turistas". Por lo que uno de los principales retos pasaría por fidelizar a sus visitantes e intentar que pernocten en el destino, generando de esta forma un gasto turístico superior así como una mayor demanda de oferta complementaria. Consideramos también las principales motivaciones de viaje, el comportamiento turístico y las valoraciones del destino según el perfil sociodemográfico (Moreira \& Galindo, 2015).

El presente artículo pretende establecer los patrones de consumo de alimentos del turista nacional que vacaciona en Mazatlán, Sinaloa, durante la Semana Santa, lo cual incluye las predilecciones gastronómicas, los restaurantes y el gasto que esto representa. El análisis se realiza con base en un conjunto de variables cuantitativas, obtenidas a través de la encuesta como instrumento de medición. A la luz de los resultados se describe la importancia de la gastronomía como parte de la oferta que brinda el destino a sus visitantes, a través del disfrute de las tradiciones culinarias del puerto, entre las que sobresalen los alimentos elaborado a base de pescados y mariscos (Gamboa \& Solorzano, 2013).

Se analiza el valor percibido como un constructo multidimensional, que involucra otras dimensiones que generan beneficios positivos de valor en el consumidor turístico y no sólo la utilidad económica. El objetivo es relacionar el perfil del turista nacional durante su experiencia en el destino turístico, con el valor percibido de sus atributos. El método cuantitativo utiliza la encuesta a turistas, con análisis factorial y ANOVA se contrasta la hipótesis. Los resultados permiten considerar aspectos relacionados con la gestión y la promoción del destino turístico, a partir de la segmentación de la demanda. Uno de los hallazgos indica que el factor del personal representa la dimensión más relevante para los turistas. Limitaciones del estudio como ser de corte transversal y la no aleatoriedad de la muestra impiden la generalización de los resultados (Solís et al., 2016). 


\section{METODOLOGÍA}

Para el desarrollo de esta investigación se empleó un método descriptivo, utilizando el instrumento metodológico de tipo encuesta, la cual se aplicó a los pobladores de la parroquia con una muestra de 395 personas. Además se realizaron visitas de campo, estas técnicas sirvieron de aporte para la obtención de información veraz y resultados positivos en el proceso de la investigación y se recurrió a una metodología bibliográfica profunda, mediante una indagación del marco teórico conceptual de los recursos naturales y desarrollo turístico tomando como bases información de revistas científicas digitales.

\section{RESULTADOS}

La población total del cantón Pasaje consta de 30.385 personas que son parte de la población económicamente activa y de la cual se obtuvo una muestra de 395 personas a las cuales se les aplicó una encuesta, de las personas encuestadas, 220 fueron de género femenino y 175 de género masculino; el $58 \%$ de los encuestados tienen la edad de 22 años, el $25 \%$ consta de 44 años mientras que el $17 \%$ tiene 54 años de edad.

El motivo principal por el cual las personas visitan Pasaje con un $29 \%$ es para visitar a sus familiares, un $21 \%$ lo hacen por diversión y un $17 \%$ por descanso y placer; siendo éstos los resultados más altos se puede decir que el lugar es muy visitado ya sea por las diferentes razones, y eso es bueno ya que se puede ir inculcando a los individuos muchos más motivos para su visita. Es por ello que Beltrán (2014) señala que el estudio de las motivaciones permite entender mejor las expectativas, las necesidades y los objetivos de los turistas y es un elemento fundamental para diseñar estrategias de marketing y ayudar al posicionamiento y competitividad de los agentes implicados en la actividad turística (Carvache et al., 2017).

La mayoría de personas manifiestan que un $39 \%$ visitan a familiares y amigos, un $18 \%$ lo hacen por recomendaciones y un $16 \%$ por cercanía del lugar de origen; es decir, que los visitantes desconocen de algunas actividades y lugares que ofrece el cantón Pasaje. Por ese motivo Martínez (2011) señala que para realizar un análisis del consumidor en cuanto a su comportamiento, es necesario diferenciar los factores externos de los internos, los factores internos son aquellos que dependen del consumidor, como la personalidad, actitudes, creencias, y entre los factores externos se destacan la cultura y estilos de vida, lo que parecería indicar que en la visita a un destino y su retorno depende no únicamente de sus atractivos o de sus servicios y su infraestructura, sino que existe una influencia de otros factores (Carvache et al., 2017).

El $83 \%$ de las personas conocen del cantón Pasaje por medios de sus amistades, un $11 \%$ por publicidad y un $6 \%$ por el Internet. Esta pregunta muestra claramente que a Pasaje le falta publicidad por todos los medios de comunicación, esto es un factor realmente importante ya que si no se fomenta el sitio las personas no podrán visitar los diferentes atractivos que se ofrece. Por tal razón Crompton et al. (2007) declaran firmemente la importancia de la comunicación para la promoción de los destinos turísticos, ya que permite estimular el interés de los turistas para que los conozcan y recuerden su existencia y atractivo (Gracia \& Torres, 2015).

Un 72\% de las personas llegan al cantón en automóvil, mientras que el $28 \%$ lo hacen en autobús, esto se da por el poco conocimiento que los individuos tienen sobre las diferentes empresas que ofrecen transporte, también manifiestan que lo hacen por seguridad y comodidad, es por ello que Bravo (1966) muestra que el transporte constituye uno de los capítulos más importantes de la oferta turística de cualquier nación, es lógico suponer que los turistas englobados en cada uno de los apartados señalados tengan distinta preferencia por los diversos medios de transporte y, por consiguiente, la evolución de la demanda de estos medios estará, en gran parte, condicionada por la estructura del turismo en el país que se considere.

Un $43 \%$ les gustaría visitar balnearios, $40 \%$ cascadas y el $17 \%$ la comunidad de Pasaje; estos resultados dan a entender que las personas sí están interesadas en visitar los diferentes lugares de recreación que cuenta el cantón, para que sus recursos sean muy bien aprovechados. Por tal razón Añon (2000) dice que la versatilidad de los balnearios, establecimientos turísticos y terapéuticos simultáneamente, posibilita el relanzamiento de este producto turístico, que constituye un potencial económico de primer orden en un sector con elevada capacidad de generar empleo y renta (López, 2003).

Un $84 \%$ si les gustaría realizar cualquier tipo de deporte extremo mientras que un $16 \%$ no, es decir que sí se podría aprovechar las diferentes áreas del cantón para la realización de las diferentes actividades, ya que existe una gran 
demanda de personas que lo harían. Esto favorece a que se fomente el turismo de aventura. Es por ello que Beltrán (2008) señala conceptualmente que el turismo de aventura se ha identificado como "viajes que tienen como fin el realizar actividades recreativas y deportivas, asociadas a desafíos impuestos por la naturaleza”. En esta novedosa actividad se expresa esa necesidad natural de las personas de experimentar sensaciones emocionantes, que evidencien una actividad física representativa bajo un esquema de recreación, con un ánimo competitivo para practicar un deporte fuera de su lugar de residencia o con el propósito de renovación y relajación.

Un $98 \%$ de los encuestados si les gustaría degustar de la gastronomía del cantón, mientras que un $2 \%$ no; siendo si el porcentaje más alto, se puede dar a conocer los platos típicos del sitio, aprovechando que la materia prima se da mismo en el lugar, es por ello que Hall et al. (2003) señala consecuentemente se puede definir al turismo gastronómico como la visita a productores primarios y secundarios de alimentos, festivales gastronómicos, restaurantes y lugares específicos donde la degustación de platos y/o la experimentación de los atributos de una región especializada en la producción de alimentos es la razón principal para la realización de un viaje (Schlüter \& Thiel, 2008):

Un $43 \%$ utilizaría un automóvil privado, un 16\% autobús urbano y un $13 \%$ motocicleta, siendo éstos los resultados más altos, se puede hacer convenios o alianzas con medios de transportes que den diferentes clases de servicio, para poder satisfacer los diferentes gustos o preferencias de los turistas. Es por ello que Casamayor (1978) indica que el transporte por carretera el principal animador del movimiento turístico mundial. La evolución del mismo ha sido creciente con una notable espectacularidad debida principalmente a los incrementos habidos en cifras absolutas, ya que ha venido a protagonizar el despegue turístico de un buen número de países.

Un 95\% sí está dispuesto a comprar un paquete turístico mientras que el $5 \%$ no, es decir que si sería factible la venta de diferentes paquetes turísticos para promover y fomentar los recursos naturales existentes aprovechándolos de una buena manera. Es por ello que Alegre et al. (2006) enseña que los diversos atributos de los paquetes turísticos son la fuente de información para que los consumidores, conforme a sus preferencias, elijan su destino para vacacionar. El paquete turístico no es más que la suma de varios servicios turísticos que son ofrecidos al cliente me- diante un precio único cerrado, no pudiéndose disgregar ni comercializar por servicio individual (Palud, 2014).

Un 78\% estaría dispuesto a pagar 25 dólares, mientras que un $22 \%$ pagaría 35 dólares, este resultado quiere decir que los precios dados son cómodos para los turistas que deseen conocer más el cantón. Es por ello que Alegre at al. (2006) indica que la estructura de precios de las ofertas puede incentivar la adquisición de paquetes con un mayor número de servicios contratados, con precios más ventajosos que otras ofertas con menores servicios. No obstante, lo cierto es que cuanto mayor sea el número de servicios contratados en un paquete, menor será el gasto realizado directamente en el destino (Palud, 2014).

\section{DISCUSIÓN Y CONCLUSIONES}

En base de los resultados obtenidos sobre la percepción del turista sobre el cantón Pasaje, Provincia de El Oro (Ecuador), el principal motivo por el cual las personas visitan el sitio es para pasar un momento agradable con su familia; es decir, que si el cantón obtuviera más publicidad en los medios de comunicación sería muy frecuentada y sus recursos naturales, culturales y patrimoniales serian conocidos y explotados con responsabilidad. El cantón Pasaje cuenta con una gran variedad de recursos y atractivos turísticos los cuales no han sido explotados del todo, uno de los recursos más utilizados por la población, es la de los ríos el cual se puede aprovechar de buena manera sin perjudicar a la naturaleza y comunidad existente.

Entre los atractivos turísticos está el río la Cocha, Casacay, Pailones del sitio Muyuyacu, Tres Cerritos, entre otros lugares. Es por eso que el caso de Pasaje está enfocado a los recursos naturales, los atractivos y el turismo ya que esta modalidad es la nueva tendencia a nivel mundial y éstas vienen de la mano para poder ayudar al buen desarrollo de una zona.

El cantón Pasaje posee tantos recursos como atractivos turísticos, la percepción del turista es positiva hacia el lugar, es decir que el principal problema es la falta de publicidad que tiene el sitio. El escaso conocimiento de la población de pasaje sobre el buen aprovechamiento de los atractivos turísticos, ha hecho que el turismo no progrese en la zona, dando esto como efecto negativo la falta de empleos y el desaprovechamiento de algunos recursos naturales nativos del cantón. El rio la Cocha es uno de los principales motivos por el cual los turistas visitaban el sitio, pero debido al mal aprovechamiento del mismo, 
los visitantes buscaron otras alternativas, hoy en día pasaje también cuenta con complejos turísticos muy bien estructurados, pero con poco personal capacitado para la atención y manejo adecuado de los atractivos turísticos. Las autoridades encargadas como el municipio no realizan de forma continua publicidad sobre el cantón y lo que posee, por eso que las personas no encuentran otro motivo más que no sea la visita a familiares para ir a Pasaje.

\section{REFERENCIAS}

Albaladejo, I., Díaz, M. (2003). Un modelo de elección discreta en la determinación del perfil del turista rural: Una aplicación a Murcia. Cuadernos de Turismo 11, 7-19.

Alvarez M. (2012). El perfil del turismo en Andalucía. eXtoikos 7, 97-100.

Anaya, J.S., Pelafox, A. (2010). El perfil del turista internacional de Cozumel a partir de la construcción de su capital simbólico. Teoría y Praxis 2, 171-185.

Barles, M., Garcés, S., Mur, M. (2012). Perfil del turista de festivales: El caso del festival internacional de las culturas Pirineo Sur. Cuadernos de Turismo 30, 63-90.

Beltrán, L., Bravo, A. (2008). Turismo de aventura: Reflexiones sobre su desarrollo y potencialidad en Colombia. Turismo y Sociedad 9, 103-112.

Bustos, R. (2001). Identidad, turismo y territorios locales. La permanente construcción. Aportes y Transferencias 5, 1, 11-28.

Carvache, W., Torres, M., Carvache, M. (2017). Análisis del perfil y satisfacción del turista que visita Montañita, Ecuador. Cuadernos de Turismo 39, 113-129.

Corral, V., Mejia, A., Atamaenda, L. (2016). Perfil del turista que visita los emprendimientos de turismo de naturaleza en la ciudad de Puyo, provincia de Pastaza-Ecuador. Revista Interamericana de Ambiente y Turismo 12, 1, 37-47.

Decasper, S.M. (2015). Competitividad y desarrollo sostenible en el sector turístico. Anais Brasileiros de Estudos Turísticos 5, 1, 48-58.

Escalona, M., Peña, F., Hiriarte, R. (2012). Oferta turística y perfil sociocultural de los empresarios turísticos del borde costero de la Araucanía, Chile. Estudios y Perspectivas en Turismo 21, 2, 306-321.

Gamboa, S., Solorzano, C. (2013). Perfil del turista nacional que consume alimentos durante Semana Santa en Mazatlán, Sinaloa. Teoría y Praxis 13, 59-81.

Gracia, G., Torres, P. (2016). El impacto de la comunica- ción pública en el desarrollo del turismo interno en el Ecuador. Alternativas 16, 2, 42-46.

Hunziker, W., Krapf, K. (s.f.). El turismo conceptos y definiciones e importancia actual. Murcia, España. Universidad de Murcia, Escuela Universitaria de Turismo de Murcia. Accesado el 4 de mayo de 2018. Disponible en http://www.interviajes.com.py/ad$\mathrm{min} / \mathrm{img} / \mathrm{pdfs} /$ turismo1c-1q4.pdf

Lavín, J., Martínez, C., Medina, F., Viteri, F. (2017). Diferencias entre el perfil del turista cultural y el turista religioso. La festividad del Señor del Terremoto en Patate (Ecuador). Revista de Ciencias Sociales 5, 1, 142-154.

López, T., Rodríguez, J., Vieria, A. (2012). Análisis diferenciado del perfil y de la motivación del turista nacional y extranjero en la ruta del vino del Marco de Jerez. Investigaciones Turísticas 6, 83-100.

López, M. (2003). El potencial turístico de los balnearios. Estudios Turísticos 157, 125-145.

Losada, M., Alen, M., Domínguez, T. (2014). Aproximación al perfil y comortamiento del turista senior. Revista Galega de Economía 23, 2, 69-89.

Moreira, P., Galindo, N. (2015). Perfil del turista cultural en ciudades patrimoniales. Los casos de San Cristóbal de la Laguna y Córdoba (España). International Journal of Scientific Management and Tourism 2, 217-229.

Palud, M.C. (2014). Valoración de atributos de los paquetes turísticos nacionales. Ciencias Económicas 11, 2, 25-42.

Pérez Redondo, J. (2017). Un viaje desde lo imaginario a lo real. Una aproximación al perfil del turista literario en España. Revista de Ciencias Sociales 5, 1, 119-129.

Realpe, F., Benítez, N. (2015). Perfil del turista que ocupa la planta hotelera de Otavalo, provincia de Imbabura, Ecuador. Interamericana de Ambiente y Turismo 11, 2, 105-135.

Romero, G. (2015). Análisis de la oferta y la demanda del turismo receptivo en el cantón Pasaje, provincia de El Oro. Universidad Técnica de Machala, Ecuador.

Schlüter, R., Thiel, D. (2008). Gastronomía y turismo en Argentina polo gastronómico Tomás Jofré. PASOS Revista de Turismo y Patrimonio Cultural 6, 2, 249-268.

Solís-Radilla, M., Hernández-Lobato, L., Villagómez-Méndez, J. (2016). El valor percibido del destino turístico en relación con el perfil del turista en Acapulco, Guerrero-México. Investigación Administrativa $46,118,1-28$. 